\title{
Impact of Particle Diameter, Particle Density and Degree of Filling on the Flow Behavior of Solid Particle Mixtures in a Rotating Drum
}

\author{
Sumudu Karunarathne $^{1} \quad$ Chameera Jayarathna $^{2}$ Lars-Andre Tokheim ${ }^{1}$ \\ ${ }^{1}$ Department of Process, Energy and Environmental Technology, University College of Southeast Norway, Norway \\ \{sumudu.karunarathne, lars.a.tokheim\}@usn. no \\ ${ }^{2}$ Tel-Tek, Norway, chameera.jayarathnaetel-tek.no
}

\begin{abstract}
Two-dimensional CFD simulations were performed to investigate the impact of density, particle diameter and degree of filling on the flow behavior of a solid particle mixture in a transverse plane of a rotary drum. The Eulerian approach with the kinetic theory of granular flow was used to simulate granular phases of $\mathrm{CaCO}_{3}$ and $\mathrm{Al}_{2} \mathrm{O}_{3}$ under the rolling mode. The volume fractions of each phase reveal that, under the considered conditions, the particle size has a greater impact on segregation than the density. Larger particles are collected at the bottom of the rotating drum while smaller particles move more into the mid-section of the bed. The active layer is responsible for the segregation owing to the trajectory mechanism. Particle segregation due to percolation is more dominant than segregation due to condensation. In addition to that, solids volume fraction variations in the moving bed indicate that the influence of the degree of particle filling made no significant impact on the degree of mixing in the rotating drum.
\end{abstract}

Keywords: rotating drums, segregation, granular flow, rolling mode, active layer

\section{Introduction}

Granular particles are processed in many ways, and for various purposes, in the industry. Rotary drums are widely used in kilns, mixers, dryers and reactors (Ding et al., 2001). In pyro-processing of materials, rotary kilns are used due to its effective mixing and heat transfer capabilities. Transverse and longitudinal mixing occur in rotating drums. In the transverse motion, six types of particle bed behavior are observed, depending on Froude number, filling degree, wall friction coefficient, ratio of particle to cylinder diameter, angle of internal friction and dynamic angles of repose (Yin et al., 2014). The bed motions are known as slipping, slumping, rolling, cascading, cataracting and centrifuging, and the rolling mode is preferred in both horizontal drums and kiln operation due to efficient mixing performance (Demagh et al., 2012; Boateng et al., 2008).

Homogeneous mixtures are required in most industries to maintain the quality of the end product. However, during processing particles may segregate when they are subjected to motion. Segregation in process equipment can be seen in many industrial applications owing to variations in the characteristics of the particles that are processed. Different particle size, shape, density, roughness and resilience are the main causes of the segregation (Boateng and Barr, 1996; Henein et al., 1983). In rotating drums, segregation can be observed in both radial and longitudinal directions. Trajectory segregation is possible in the flow regimes of slumping, rolling and cataracting, in which finer particles will be concentrated in the mid-chord section (Boateng and Barr, 1996). Percolation and condensation are two other segregation mechanisms that may occur in rotating drums. Percolation is caused by differences in particle size, whereas condensation is due to variation in particle densities. One of these mechanisms may dominate in the active layer of the moving bed, leading to segregation of particles during drum rotation (Chen et al., 2016).

Several experimental and numerical simulations have been done to investigate particle dynamics in rotating drums. Boateng and Barr (1996) developed a mathematical model to investigate mixing and segregation in a transverse plane of a rotary kiln. Their model was capable of predicting the active layer depth and the velocity distribution. The discrete element method (DEM) is a promising technique to investigate particle motion in many industrial applications. Longitudinal and transverse mixing in rotary kilns were studied by Finnie et al. (2005) using the DEM approach. Mixing in the longitudinal direction was explained by a one dimensional diffusion equation, and transverse mixing was determined by using an "entropy" like quantity proposed by Schutyser et al. (2001). Chen et al. (2016) performed a numerical simulation to investigate radial mixing and segregation of a granular bed considering particle size and density variations. It was concluded that a homogeneous mixture can be obtained by achieving an equilibrium state between percolation and condensation by controlling the volume ratio or the density ratio of the granular particles.

This study focuses on two-dimensional (2D) numerical simulations of mixing and segregation of two granular phases in a rotating drum. Two granular materials, calcium carbonate $\left(\mathrm{CaCO}_{3}\right)$ and aluminum oxide $\mathrm{Al}_{2} \mathrm{O}_{3}$, were considered in the simulations. The Eulerian approach along with the kinetic theory of granular flow were used to analyze the particle dynamics and segregation in the transverse plane. The focus was on understanding how density, particle size 
and degree of filling affect the particle segregation in a rotating drum.

A two-dimensional geometry was created to mimic the characteristics of the transverse plane of a rotating drum. The numerical calculations were performed by the CFD software ANSYS FLUENT 16.2.

\section{Model Description}

The Eulerian approach is applicable to flows with $\mathrm{N}$ number of phases. Here, the governing equations for the Euler-Euler model and the kinetic theory of granular flow are discussed for a solid mixture.

\subsection{Governing Equations in the Euler-Euler Method}

\subsubsection{Continuity Equations}

Conservation of mass in the flow is represented by continuity equations for the gas phase and the solid phases,

$$
\begin{aligned}
& \frac{\partial}{\partial t}\left(\varepsilon_{g} \rho_{g}\right)+\nabla \cdot\left(\varepsilon_{g} \rho_{g} v_{g}\right)=0 \\
& \frac{\partial}{\partial t}\left(\varepsilon_{S} \rho_{S}\right)+\nabla \cdot\left(\varepsilon_{S} \rho_{S} v_{S}\right)=0
\end{aligned}
$$

Here, $\rho$ is density, $v$ is velocity, $\varepsilon$ is volume fraction and $t$ is time. $S$ and $g$ refer to the solids phase and the gas phase, respectively.

\subsubsection{Momentum Equations}

The influence of viscous, pressure and gravity forces on the dynamics of the gas and the solid particles is described by momentum equations. The momentum equations for the gas phase and the solid phases are written as (Demagh et al., 2012; Azadi, 2011):

$$
\begin{aligned}
\frac{\partial}{\partial t}\left(\varepsilon_{g} \rho_{g} v_{g}\right)+\nabla \cdot\left(\varepsilon_{g} \rho_{g} v_{g} v_{g}\right)= & -\varepsilon_{g} \nabla P_{g}+\varepsilon_{g} \rho_{g} g \\
& -\sum_{s=1}^{N} k_{g s}\left(v_{g}-v_{s}\right)+\nabla \cdot\left(\varepsilon_{g} \tau_{g}\right) \\
\frac{\partial}{\partial t}\left(\varepsilon_{s} \rho_{s} v_{s}\right)+\nabla \cdot\left(\varepsilon_{s} \rho_{s} v_{s} v_{s}\right)= & -\varepsilon_{s} \nabla P_{g}+\varepsilon_{s} \rho_{s} g+k_{g s}\left(v_{g}-v_{s}\right) \\
& +\sum_{n=1, s \neq n}^{N} k_{n s}\left(v_{n}-v_{s}\right)+\nabla \tau_{s}
\end{aligned}
$$

Here, $P_{g}, k_{g s}, k_{n s}, \tau_{g}$ and $g$ are the fluid pressure, gassolid momentum exchange coefficient between the gaseous and solids phases, solid-solid momentum exchange coefficient, the viscous stress tensor of the gas phase and the gravity constant, respectively.
The Newtonian form of the viscous stress tensor for the gas phase, $\tau_{g}$ in Eq (3), and for the solids phase, $\tau_{s}$ in Eq (4), are given by (Liu et al., 2016):

$$
\begin{gathered}
\tau_{g}=v_{g}\left[\nabla v_{g}+\left(\nabla v_{g}\right)^{T}-\frac{2}{3} \mu_{g}\left(\nabla \cdot v_{g}\right)\right] I \\
\tau_{s}=\left(-P_{s}+\zeta_{s} \nabla \cdot v_{s}\right) I+\mu_{s}\left\{\left[\nabla v_{s}+\left(\nabla v_{s}\right)^{T}\right]-\frac{2}{3}\left(\nabla \cdot v_{s}\right) I\right\}
\end{gathered}
$$

Here $P_{s}, \mu_{s}, \zeta_{s}$ and $I$ are the solids pressure, the solids viscosity, the solids bulk viscosity and the unit tensor, respectively.

$P_{s}$ represents the solid pressure (normal forces) created due to particle-particle collisions in a flow due to presence of several solid phases (Gidaspow, 1994)

$$
P_{s}=\varepsilon_{s} \rho_{s} \Theta_{s}+\sum_{n=1}^{N} 2 \frac{d_{n s}^{3}}{d_{s}^{3}}\left(1+e_{n s}\right) g_{0, n s} \varepsilon_{n} \varepsilon_{s} \rho_{s} \Theta_{s}
$$

$e_{s n}$ is the particle-particle restitution coefficient between phase $s$ and $n . d_{s}$ is the particle diameter. $d_{n s}$ is the mean diameter of the particles in phase $n$ and s. $g_{o, n s}$ and $\Theta_{s}$ are the radial distribution function and the granular temperature respectively.

The bulk viscosity of the solids, $\zeta_{s}$ in $\mathrm{Eq}(6)$, is given by (Neri and Gidaspow, 2000):

$$
\zeta_{s}=\frac{4}{3} \varepsilon_{s}^{2} \rho_{s} d_{s} g_{0, s s}\left(1+e_{s s}\right) \sqrt{\frac{\Theta_{s}}{\pi}}
$$

The solids shear viscosity in Eq (6) is given as (Arastoopour, 2001):

$\mu_{s}=\frac{4}{5} \varepsilon_{s}^{2} \rho_{s} d_{s} g_{0, s s}\left(1+e_{s s}\right) \sqrt{\frac{\Theta_{s}}{\pi}}+\frac{10 \rho_{s} d_{s} \sqrt{\pi \Theta_{s}}}{96\left(1+e_{s s}\right) \varepsilon_{s} g_{0, s s}}\left[1+\frac{4}{5} \varepsilon_{s} g_{0, s s}\left(1+e_{s s}\right)\right]^{2}$

Wen and Ergun (Huilin and Gidaspow, 2003) proposed that the exchange coefficient $k_{g s}$ between the gas and the solids phase given in Eq (4) and (5) could be calculated by:

$$
\begin{aligned}
& \left.k_{g s}\right|_{\text {Wen \& Yu }}=\frac{3}{4} C_{D} \frac{\rho_{s} \varepsilon_{s}\left|v_{g}-v_{s}\right|}{d_{s}} \varepsilon_{g}{ }^{-2.65} \quad \varepsilon_{g}>0.8 \\
& \left.k_{g s}\right|_{\text {Ergun }}=150 \frac{\left(1-\varepsilon_{g}\right) \varepsilon_{s} \mu_{g}}{\left(\varepsilon_{g} d_{s}\right)^{2}}+1.75 \frac{\rho_{g} \varepsilon_{s}\left|v_{g}-v_{s}\right|}{\varepsilon_{s} d_{s}} \quad \varepsilon_{g} \leq 0.8
\end{aligned}
$$

The drag coefficient depends on the value of the Reynolds number, Re:

$$
\begin{cases}C_{D}=\frac{24}{\operatorname{Re}}\left(1+0.15 \mathrm{Re}^{0.687}\right) & \mathrm{Re}<1000 \\ C_{D}=0.44 & \mathrm{Re} \geq 1000\end{cases}
$$

$$
\operatorname{Re}=\frac{\rho_{g} \varepsilon_{g}\left|v_{g}-v_{s}\right| d_{s}}{\mu_{g}}
$$




\subsubsection{Kinetic Theory of Granular Flow}

The continuum approach of granular flow requires a method to calculate viscous forces applied on the solid particles. Particle collisions are considered to predict the physical properties of the particulate phase. The theory is widely used in modelling of particulate systems to simulate actual systems with a high level of accuracy.

The kinetic theory introduces a new variable $\Theta$, called granular temperature, and it is a measure of the kinetic energy of the solids. One-third of the mean square velocity of the random motion of the particles is considered as the granular temperature, $\Theta=v_{s}^{\prime 2} / 3$, where $v_{s}^{\prime 2}$ is the square of the fluctuating velocity of the particle. A transport equation for the granular temperature can be written as (Huilin et al., 2001):

$$
\begin{aligned}
\frac{3}{2}\left[\frac{\partial}{\partial t}\left(\varepsilon_{s} \rho_{s} \Theta_{s}\right)+\nabla \cdot\left(\varepsilon_{s} \rho_{s} \Theta_{s}\right) v_{s}\right] & =\left(\nabla P I+\varepsilon_{s} \nabla \tau_{s}\right): \nabla v_{s}+\nabla \cdot\left(k_{s} \nabla \Theta_{s}\right)-\gamma_{s} \\
& +\Phi_{s}+D_{g s}
\end{aligned}
$$

Here, $\gamma_{s}$ is dissipation of turbulent kinetic energy, $\Phi_{s}$ is energy exchange between gas and particle and $D_{g s}$ is energy dissipation.

The turbulent kinetic energy dissipation, $\gamma_{s}$ in Eq (14), is given as (Neri and Gidaspow, 2000):

$$
\gamma_{s}=3\left(1-e_{s s}^{2}\right) \varepsilon_{s}^{2} \rho_{s} g_{0, s s} \Theta_{s}\left(\frac{4}{d_{s}} \sqrt{\frac{\Theta_{s}}{\pi}}-\nabla \cdot v_{s}\right)
$$

The radial distribution for $\mathrm{N}$ solid phases can be expressed as (Ahmadi and Ma, 1990):

$$
\begin{gathered}
g_{o, s s}=\frac{1+2.5 \varepsilon_{s}+4.59 \varepsilon_{s}^{2}+4.52 \varepsilon_{s}^{3}}{\left(1-\left(\frac{\varepsilon_{s}}{\varepsilon_{s, \max }}\right)^{3}\right)^{0.678}}+\frac{1}{2} d_{s} \sum_{n=1}^{N} \frac{\varepsilon_{n}}{d_{n}} \\
\varepsilon_{s}=\sum_{n=1}^{N} \varepsilon_{n}
\end{gathered}
$$

$n$ are solid phases only, and $d_{s}$ is the diameter of a particle in the $s^{\text {th }}$ phase.

The energy exchange between the gas and the solids phases in Eq (14) is defined as:

$$
\Phi_{s}=-3 k_{g s} \Theta_{s}
$$

The rate of energy dissipation per unit volume is expressed in the following equation:

$$
D_{g s}=\frac{d_{s} \rho_{s}}{4 \sqrt{\pi \Theta_{s}}}\left(\frac{18 \mu_{g}}{d_{s}^{2} \rho_{s}}\right)^{2}\left|v_{g}-v_{s}\right|^{2}
$$

\subsection{Simulation}

The simulations were performed under a Froude number of $16 \times 10^{-4}$ to maintain the rolling mode in the rotating drum. The cylinder and the particles rotate in the counterclockwise direction. The drum was simulated with different values for the degree of filling: 10, 15 and $20 \%$ of the drum height.

\subsubsection{Physical Properties of Materials and Model Parameters}

Two granular phases of $\mathrm{CaCO}_{3}$ and $\mathrm{Al}_{2} \mathrm{O}_{3}$ were used in the simulations. The physical properties of the solids, as well as the model parameters, are given in table 1 .

Table 1. Physical Properties of Materials and Model Parameters

\begin{tabular}{|l|l|c|}
\hline Parameter & Description & Value \\
\cline { 1 - 1 }$\rho_{\mathrm{CaCO}_{3}}\left(\mathrm{~kg} / \mathrm{m}^{3}\right)$ & Particle density & 1760 \\
\cline { 1 - 1 }$\rho_{\mathrm{Al}_{2} \mathrm{O}_{3}}\left(\mathrm{~kg} / \mathrm{m}^{3}\right)$ & & 3000 \\
\cline { 1 - 1 }$d_{\mathrm{CaCO}_{3}}(\mu \mathrm{m})$ & Particle diameter & 175 \\
\cline { 1 - 1 }$d_{\mathrm{Al}_{2} \mathrm{O}_{3}}(\mu \mathrm{m})$ & & 1000 \\
\cline { 1 - 1 }$\omega(\mathrm{rpm})$ & Rotational speed & 2 \\
\hline
\end{tabular}

\subsubsection{Geometry and Mesh}

A circular geometry with a diameter of $0.4 \mathrm{~m}$ was created with a mesh of 5500 elements to represent the transverse plane of the rotating cylinder. Figure 1 shows the mesh of the transverse plane.

\subsubsection{Initial and Boundary Conditions}

In rotating drums, particles are subjected to wall friction and gravity forces. A proper boundary condition should be used for the relative motion between the particles and the drum wall. Here, a no-slip condition was assumed, meaning that the relative velocities of the gas and the particles at the wall are set to zero.

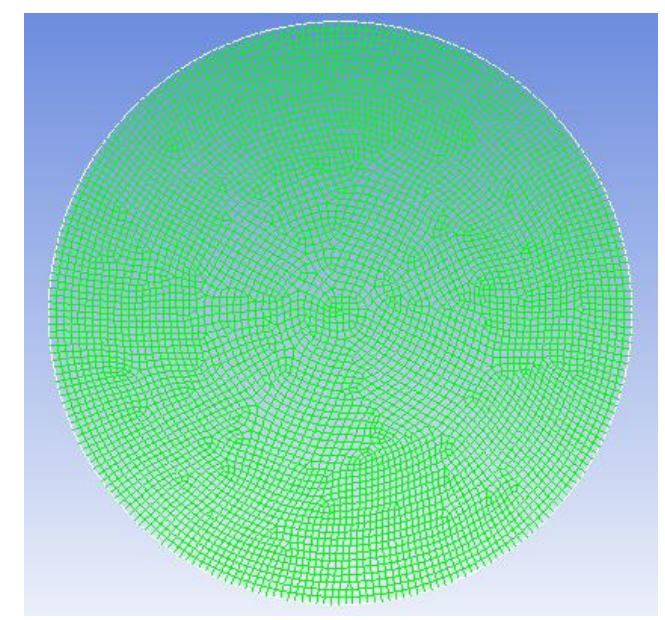

Figure 1. Mesh of the transverse plane 


\subsubsection{Solution Strategy and Convergence Criteria}

In this study, the governing equations of the model were solved using the finite volume approach. The fluids were taken as incompressible, and a pressure-based solver was used. The "SIMPLE" algorithm (Patankar and Spalding, 1972) was used for the coupling between pressure and velocity. Discretization of the governing equations was carried out according to the second order upwind scheme (Banks and Henshaw, 2012). The "QUICK" scheme (Versteeg and Malalasekera, 2007) was used to discretize the volume fractions. Finally, the time step of the simulations was $10^{-3} \mathrm{~s}$, and the residual values for convergence were set to $10^{-3}$.

\section{Results and Discussion}

In rolling mode, particles in the active layer move relatively fast in comparison with the particles in the passive layer. The distance of the particle travel in the active layer is proportional to the square of the particle diameter (Boateng and Barr, 1996). Consequently smaller particles are likely to be concentrated in the midchord section.

\subsection{Effect of Particle Size on Segregation}

The effect of particle size on segregation of granular particles was examined by considering two particles with different size but with the same density. The simulation was carried out with particle sizes of $175 \mu \mathrm{m}$ and $1000 \mu \mathrm{m}$. The density of the particles was the same as the density of $\mathrm{CaCO}_{3}$. Figures 2 and 3 show a significant segregation due to particle size (percolation). The smaller particles concentrate in the mid-section of the moving bed while the larger particles sink towards the bottom of the drum.
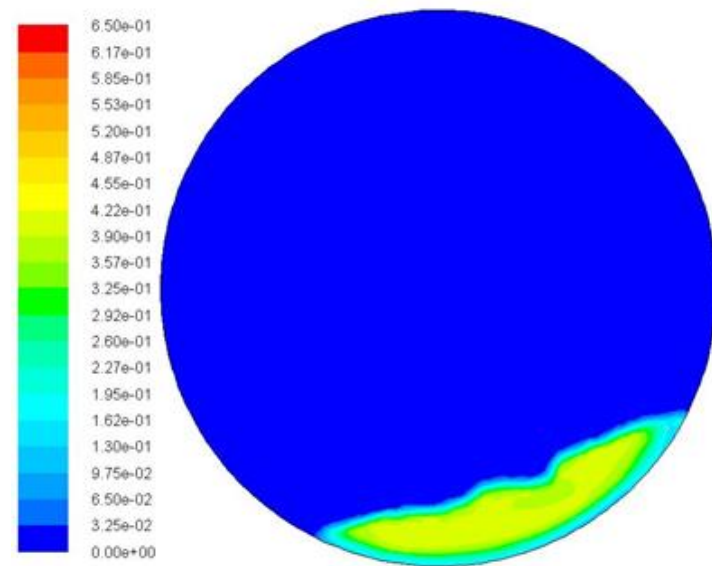

Figure 2. Volume fraction of particles with a diameter of $175 \mu \mathrm{m}$ at pseudo steady-state.

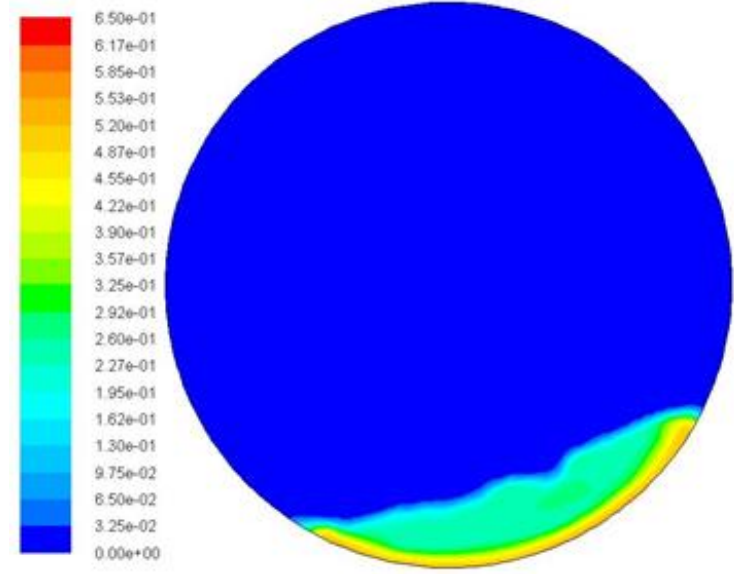

Figure 3. Volume fraction of particles with a diameter of $1000 \mu \mathrm{m}$ at pseudo steady-state.

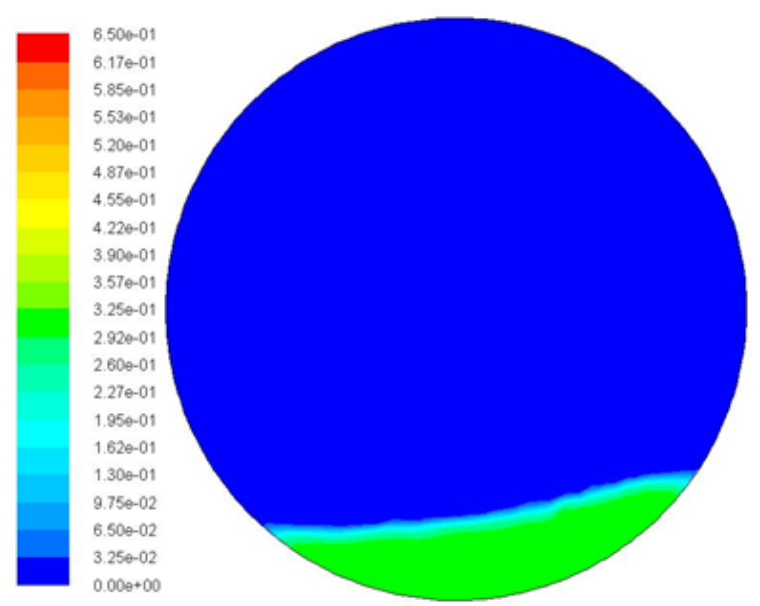

Figure 4. Volume fraction of particles with a density of $3000 \mathrm{~kg} / \mathrm{m}^{3}$ in a mixture of particles with the same particle size at pseudo steady-state

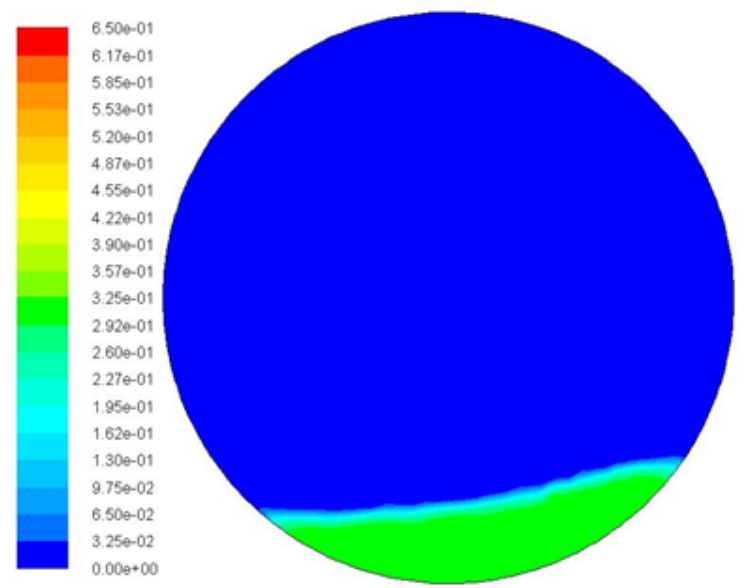

Figure 5. Volume fraction of particles with a density of $1760 \mathrm{~kg} / \mathrm{m}^{3}$ in a mixture of particles with the same particle size at pseudo steady-state 

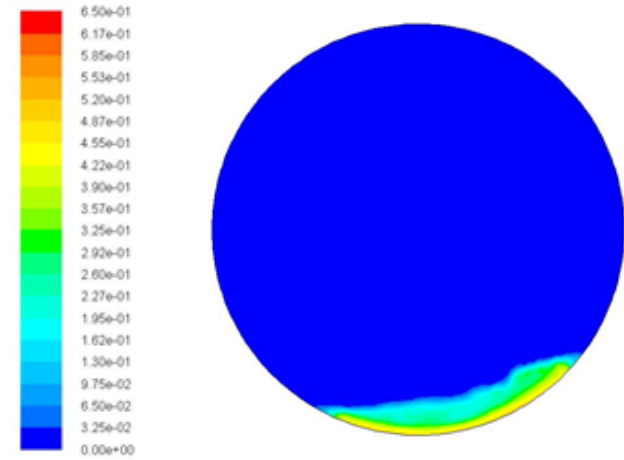

a

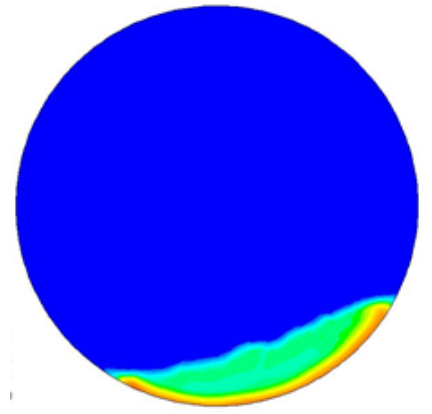

b

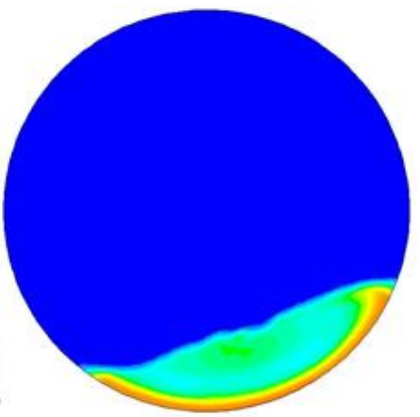

c

Figure 6. Volume fraction of $\mathrm{Al}_{2} \mathrm{O}_{3}$ at pseudo steady-state with different degrees of particle filling, (a) $10 \%$, (b) $15 \%$, (c) $20 \%$
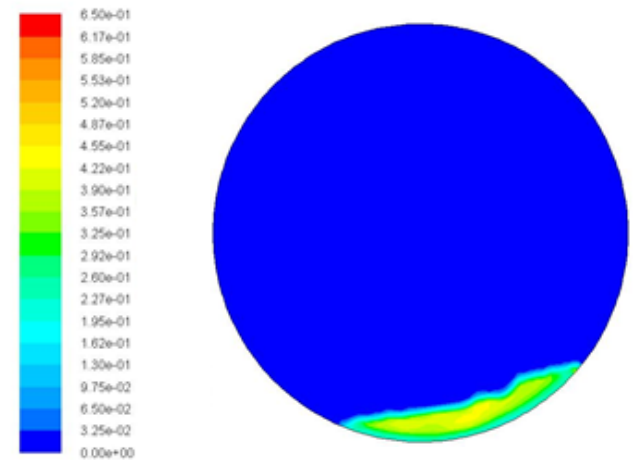

a

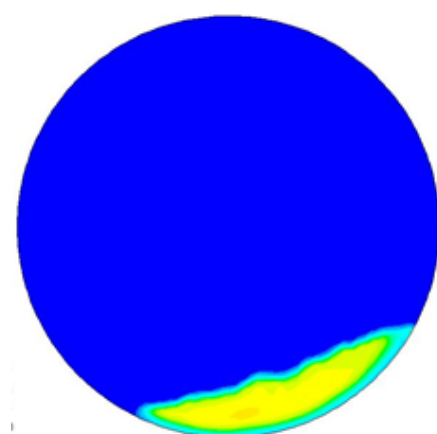

b

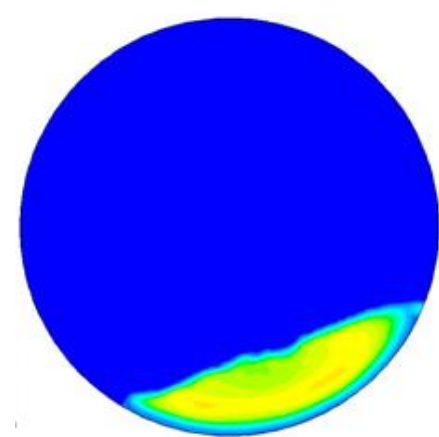

c

Figure 7. Volume fraction of $\mathrm{CaCO}_{3}$ at pseudo steady-state with different degrees of particle filling, (a) 10\%, (b) $15 \%$, (c) $20 \%$

\subsection{Effect of Density on Segregation}

Segregation due to density variations was investigated considering the same particle size with different densities. The particle size was set to $1000 \mu \mathrm{m}$ and the densities were $1760 \mathrm{~kg} / \mathrm{m}^{3}$ and $3000 \mathrm{~kg} / \mathrm{m}^{3}$. Volume fractions of the two particle types are shown in Figures 4 and 5, which show that for the particles considered no significant segregation appears due to density differences (condensation). According to Jha (2008), the particle size is the more dominant parameter for segregation, and this is in line with the present simulation results.

\subsection{Effect of Degree of Filling on Segregation}

Figures 6 and 7 show the volume fractions of $\mathrm{CaCO}_{3}$ and $\mathrm{Al}_{2} \mathrm{O}_{3}$, respectively, with different degrees of fillings. The simulation results show that, with the selected particle sizes and densities, there will be a considerable segregation in all three cases. The segregation tendency is, however, more or less the same for all three, hence the degree of filling does not make a big impact on the particle motion pattern as such.

\section{Conclusions}

Two-dimensional simulations of the transverse plane in a rotating drum, based on the Eulerian approach and the kinetic theory of granular flow, indicate that particle segregation occurs mainly due to differences in particle size when the flow is in the rolling mode. The active layer in the rolling mode constitutes an environment facilitating segregation. Trajectory segregation and percolation seem to be the predominant mechanisms in the current simulations as no significant segregation appeared with different densities, hence segregation due to condensation was less important. The degree of particle filling in the rotary drum made no significant impact on the particle behavior.

\section{References}

G. Ahmadi and D. Ma. A thermodynamical formulation for dispersed multiphase turbulent flows-1. International Journal of Multiphase Flow, 16: 323-340, 1990.

H. Arastoopour. Numerical simulation and experimental analysis of gas/solid flow systems: 1999 Fluor-Daniel Plenary lecture. Powder Technology, 119: 59-67, 2001.

M. Azadi. Multi-fluid Eulerian modeling of limestone particles elutriation from a binary mixture in a gas solid fluidized bed. Journal of Industrial and Engineering Chemistry, 17: 229-236, 2011. 
J. W. Banks and W. D. Henshaw. Upwind schemes for the wave equation in second-order form. Journal of Computational Physics, 231: 5854-5889, 2012.

A. A. Boateng, Rotary Kilns: Transport Phenomena and Transport Processes. USA: Butterworth-Heinemann publications, 2008.

A. A. Boateng and P. V. Barr. Modelling of particle mixing and segregation in the transvers plane of a rotary kiln. Chemical Engineering Science, 51: 4167-4181, 1996.

H. Chen, X. Zhao, Y. Xiao, Y. Liu, and Y. Liu. Radial mixing and segregation of granular bed bi-dispersed both in particle size and density within horizontal rotating drum. Transactions of Nonferrous Metals Society of China, 26: 527535, 2016.

Y. Demagh, Hocine, B. Moussa, M. Lachi, and L. Bordja. Surface particle motion in rotating cylinders: Validation and similarity for an industrial scale kiln. Powder Technology, 224: 260-272, 2012.

Y. L. Ding, J. P. K. Seville, R. Forster, and D. J. Parker. Solid motion in rolling mode rotating drums operated at low to medium rotational speeds. Chemical Engineering Science, 56: 1769-1780, 2001.

G.J.Finnie, N.P.Kruyt, M.Ye, C.Zeilstra, and J.A.M.Kuipers. Longitudinal and transverse mixing in rotary kilns: A discrete element method approach. Chemical Engineering Science, 60: 4083-4091, 2005.

D. Gidaspow, Multiphase flow and fluidization. California: Academic press, 1994.

H. Henein, J. K. Brimacombe, and A. P. Watkinson. Experimental study of transverse bed motion in rotary kiln. Metallurgical Transactions B, 14B: 191-205, 1983.

L. Huilin and D. Gidaspow. Hydrodynamics of binary fluidization in a riser: CFD simulation using two granular temperatures. Chemical Engineering Science, 58: 3777-3792, 2003.

L. Huilin, D. Gidaspow, and E. Manger. Kinetic theory of fluidized binary granular mixtures. Phys. Rev. E, 64:061301: $1-8,2001$.

A. K. Jha. Percolation segregation in multi-size and multicomponent particulate mixtures: Measurement, sampling, and modeling. PhD, Graduate School, College of Engineering Pennsylvania State University, 2008.

H. Liu, H. Yin, M. Zhang, M. Xie, and X. Xi. Numerical simulation of particle motion and heat transfer in a rotary kiln. Powder Technology, 287: 239-247, 2016.

A. Neri and D. Gidaspow. Riser hydrodynamics: Simulation using kinetic theory. AIChE Journal, 46: 52-67, 2000.

S. V. Patankar and D. B. Spalding. A calculation procedure for heat, mass and momentum transfer in three-dimensional parabolic flows. International Journal of Heat and Mass Transfer, 15: 1787-1806, 1972.

M. A. I. Schutyser, J. T. Padding, F. J. Weber, and W. J. Briels. Discrete particle simulations predicting mixing behavior of solid substrate particles in a rotating drum fermenter. Inc. Biotechnol Bioeng, 75: 666-675, 2001.

H. K. Versteeg and W. Malalasekera, An introduction to computational fluid dynamics, second ed. England: Pearson Education Limited 2007.

H. Yin, M. Zhang, and H. Liu. Numerical simulation of threedimensional unsteady granular flows in rotary kiln. Powder Technology, 253: 138-145, 2014. 